\title{
Precision neutron flux measurement using the Alpha-Gamma device
}

\author{
Evan R. Adamek ${ }^{1, a}$, Maynard S. Dewey ${ }^{2}$, Nadia Fomin ${ }^{1}$, David M. Gilliam ${ }^{2}$, Geoffrey L. Greene ${ }^{1,3}$, \\ Shannon F. Hoogerheide ${ }^{2}$, Hans P. Mumm ${ }^{2}$, Jeffrey S. Nico ${ }^{2}$, and William M. Snow ${ }^{4}$ \\ ${ }^{1}$ University of Tennessee, Knoxville, TN 37996, USA \\ 2 National Institute of Standards and Technology, Gaithersburg, MD 20899, USA \\ ${ }^{3}$ Oak Ridge National Laboratory, Oak Ridge, TN 37830, USA \\ ${ }^{4}$ Indiana University, Bloomington, IN 47408, USA
}

\begin{abstract}
The Alpha-Gamma device at the National Institute of Standards and Technology (NIST) utilizes neutron capture on a totally absorbing ${ }^{10} \mathrm{~B}$ deposit to measure the absolute neutron flux of a monochromatic cold neutron beam. Gammas produced by the boron capture are counted using high purity germanium detectors, which are calibrated using a well-measured ${ }^{239} \mathrm{Pu}$ alpha source and the alpha-to-gamma ratio from neutron capture on a thin ${ }^{10} \mathrm{~B}$ target. This device has been successfully operated and used to calibrate the neutron flux monitor for the BL2 neutron lifetime experiment at NIST. It is also being used for a measurement of the ${ }^{6} \mathrm{Li}(\mathrm{n}, \mathrm{t})^{4} \mathrm{He}$ cross section. We shall present its principle of operation along with the current and planned projects involving the Alpha-Gamma device, including the recalibration of the U.S. national neutron standard NBS-1 and $(n, f)$ cross section measurements of ${ }^{235} \mathrm{U}$.
\end{abstract}

\section{Introduction}

Precision experiments with low energy neutrons have provided extensive insight into the fundamental physics of particles and other basic and applied areas of scientific discovery. The NIST Center for Neutron Research (NCNR) is a dedicated neutron science facility which utilizes a $20 \mathrm{MW}$ heavy water moderated ${ }^{235} \mathrm{U}$ reactor to provide cold neutron beams to twenty-one research stations. In addition to its primary focus on neutron scattering experiments, the NCNR guide hall houses experiments in imaging, calibration, and fundamental neutron physics; most notably, a second run of the beam lifetime experiment, BL2, is currently underway as discussed by Hoogerheide, et al. elsewhere in these proceedings [1].

The Alpha-Gamma device installed at the monochromatic beam station NG-6M at the NCNR is an absolute neutron flux measurement device that is used in precision calibration of neutron flux monitors, including the one used in the BL2 apparatus. Flux is defined here as the quotient of the particle number incremented through an area in space within a time interval, with units $s^{-1}$. As the cross sectional area of all targets typically used on the neutron beam are designed to be larger than the neutron beam area, use of flux rather than fluence rate $(d N$ by $d A d t)$ is preferred. The principle of the device's operation has been published previously [2] and will be briefly reviewed in Sect. 2. Current measurement campaigns as well as projects planned for the immediate future will be discussed in Sect. 3.

a e-mail: eadamek@utk.edu

\section{Principle of operation}

\subsection{The Alpha-Gamma apparatus}

The Alpha-Gamma device contains a target mount at its center with two diametrically opposed high purity germanium (HPGe) gamma detectors along the vertical axis and a $900 \mathrm{~mm}^{2}$ silicon surface barrier charged particle detector coaxial with the upstream face of the target mount (see Fig. 1). The silicon detector is enclosed in a case with a $27.6 \mathrm{~mm}$ diameter circular aperture, which will henceforth be referred to as the " $\alpha$ detector". During operation, this setup is held under vacuum at a maximum pressure of $0.04 \mathrm{~Pa}$. The measurement requires calibration of the $\alpha$ detector including the solid angle $\left(\Omega_{\mathrm{AG}}\right)$ it subtends on the installed target. The target installed into the target mount alternates between a "thin" target, consisting of a $25 \mu \mathrm{g} / \mathrm{cm}^{2}$ deposit of $94.5 \%$ enriched ${ }^{10} \mathrm{~B}$ on a single crystal silicon substrate, and a "thick" $98 \%$ enriched ${ }^{10} \mathrm{~B}_{4} \mathrm{C}$ totally absorbing target.

Measurement of the neutron flux is based on the ${ }^{10} \mathrm{~B}$ capture reaction [3]. This capture produces a ${ }^{7} \mathrm{Li}$ nucleus and an $\alpha$ particle, and $93.70 \%$ of the time (defining the branching ratio as $b_{\alpha \gamma}$ ) the lithium nucleus is in an excited state which subsequently emits a gamma

$$
\begin{aligned}
n & +{ }^{10} \mathrm{~B} \rightarrow{ }^{7} \mathrm{Li}^{*}+\alpha \\
& \rightarrow{ }^{7} \mathrm{Li}(840 \mathrm{keV})+\alpha(1472 \mathrm{keV})+\gamma(478 \mathrm{keV}) .(1)
\end{aligned}
$$

The capture with no gamma emitted has a branching ratio of $b_{\alpha, 0}=6.30 \%$ and is described by

$$
n+{ }^{10} \mathrm{~B} \rightarrow{ }^{7} \operatorname{Li}(1015 \mathrm{keV})+\alpha(1776 \mathrm{keV}) .
$$

A monochromatic cold neutron beam collimated to a diameter of up to $15 \mathrm{~mm}$ is delivered via a helium-filled

(c) The Authors, published by EDP Sciences. This is an Open Access article distributed under the terms of the Creative Commons Attribution License 4.0 (http://creativecommons.org/licenses/by/4.0/). 


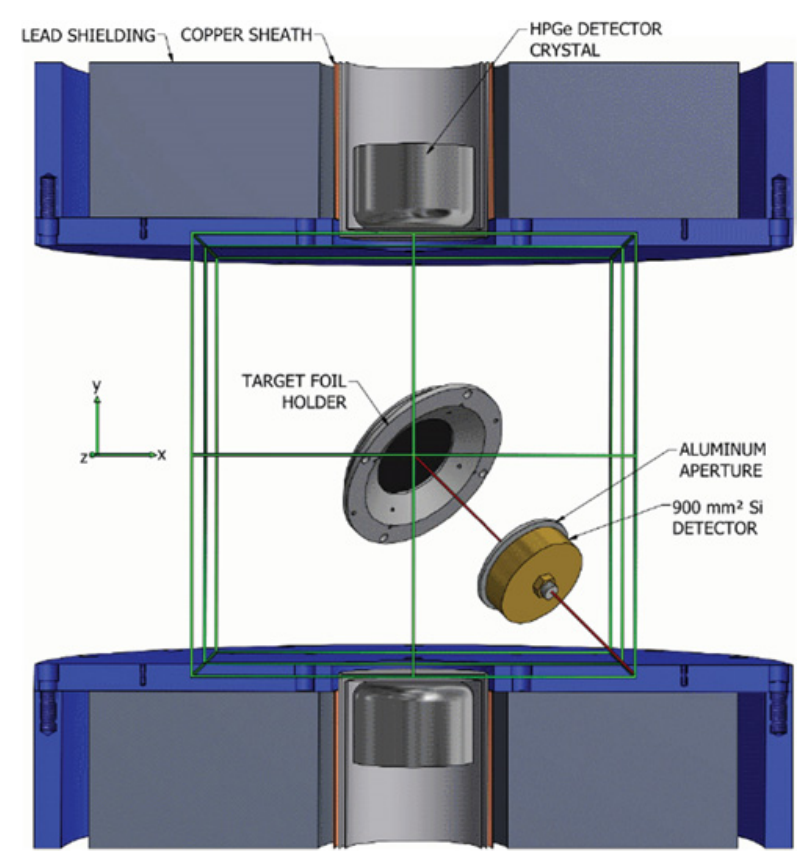

Figure 1. Layout of the Alpha-Gamma target mount showing the detectors. The neutron beam is incident from the right of the image.

flight tube to the apparatus to induce the capture reaction. By investigating the detection rates of alphas and gammas ( $r_{\alpha}$ and $r_{\gamma}$, respectively) emitted from a thin target, it is possible to derive an expression for the gamma efficiency incorporating both the solid angle and the photopeak efficiency

$$
\epsilon_{\gamma}=\frac{1}{b_{\alpha \gamma}} \frac{r_{\gamma, t h i n}}{r_{\alpha, t h i n}} \Omega_{A G}
$$

By calibrating the gamma detectors in this fashion, the thin target can be swapped for the thick target and the absolute neutron rate can be obtained

$$
R_{n}=\frac{r_{\gamma, \text { thick }}}{\epsilon_{\gamma} b_{\alpha \gamma}}=r_{\gamma, \text { thick }} \frac{r_{\alpha, \text { thin }}}{r_{\gamma, \text { thin }}} \frac{1}{\Omega_{A G}} .
$$

Note that the branching ratio cancels in this expression, and so it is not an input to the measurement. For a beam measurement, a thick measurement run is bookended by two thin measurement runs, with each run lasting about one day. For each detector, a lower and upper energy threshold is set to define a region of interest for the desired particle. As seen in Fig. 2, the windows on the HPGe detectors are set to capture the $478 \mathrm{keV}$ gamma peak while the $\alpha$ detector window is defined by its lower threshold below the pair of alpha peaks from the two capture processes (see Fig. 3). The $r_{\alpha} / r_{\gamma}$ ratio for each of the thin runs is computed from the detector count rates and interpolated to produce the effective ratio at the time of the thick measurement; this eliminates any linear drift in the gamma detector response. The thick target run represents the direct measurement of the beam given the calibration provided by the thin measurement.

In computing Eq. (3) and (4), it is necessary to know the solid angle of the $\alpha$ detector. This is determined through measurement of a well-calibrated alpha source, namely a ${ }^{239} \mathrm{Pu}$ deposit on silicon. In a separate experiment, this source is placed in a low solid angle
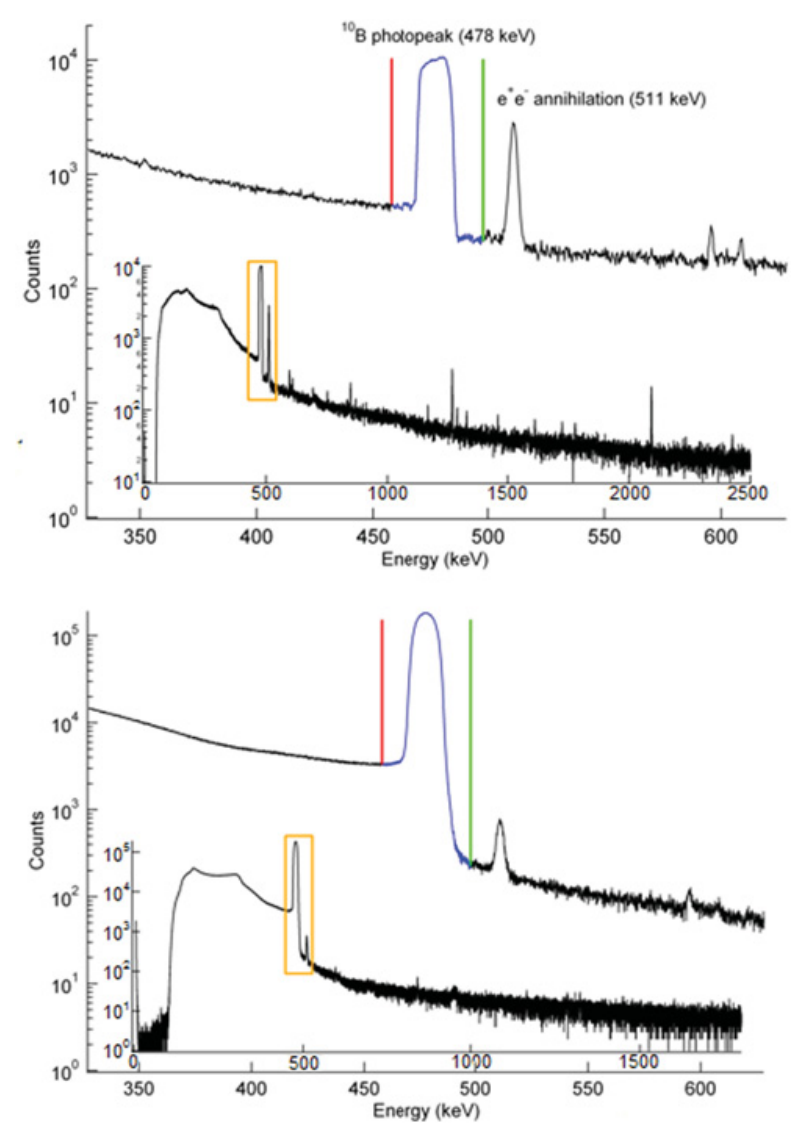

Figure 2. Spectra from the Alpha-Gamma HPGe detectors during thin target (top) and thick target (bottom) measurement runs. The boxed region of each spectrum is maginfied, with the lower and upper SCA thresholds indicated.

precision counting stack and the $\alpha$ rates are measured with an apertured surface barrier detector. The backgroundsubtracted counts within the region of interest give the emission rate of the source multiplied by the known counting stack solid angle. With the rate thus determined, the $\mathrm{Pu}$ source is installed into the apparatus and the ratio of the measured alpha rate, $r_{P u, A G}$, to the known emission rate, $R_{P u, \text { emitted }}$, determines the solid angle.

\subsection{Calibrating the BL2 flux monitor}

The absolute neutron counting that Alpha-Gamma provides is used to calibrate a flux monitor on the same beam to sub- $0.1 \%$ precision. The typical form of the flux monitor is shown in Fig. 4. Four surface barrier charged particle detectors are placed symmetrically about a target consisting of ${ }^{6} \mathrm{LiF}$ deposited on a silicon substrate that is positioned normal to the beam. The geometry is defined by a precisely measured rigid structure which provides fixed apertures in front of each detector location. The capture reaction of neutrons on lithium is

$$
n+{ }^{6} \mathrm{Li} \rightarrow \alpha(2070 \mathrm{keV})+{ }^{3} \mathrm{H}(2720 \mathrm{keV}) \text {. }
$$

The alpha and triton signals are easily identified in the spectrum. The signal rate, $r_{F M}$, is determined by summing the counts above the preset thresholds (shown in Fig. 3) for each detector, and intercomparison of these count rates provides verification of symmetric positioning. By calibrating the flux monitor on the same monochromatic beamline as Alpha-Gamma, knowledge of three factors in 

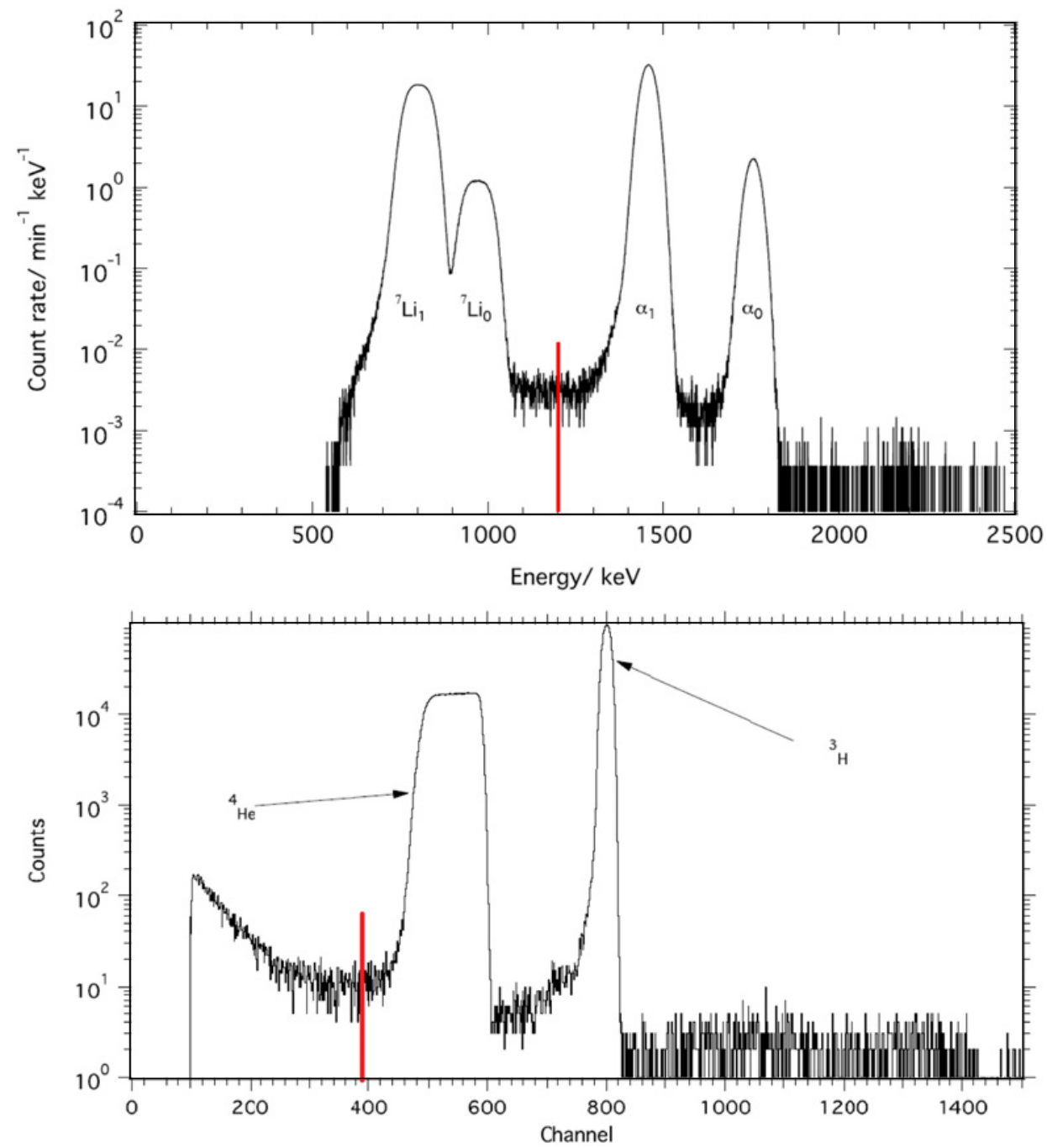

Figure 3. Spectra from the Alpha-Gamma $\alpha$ detector (top) and flux monitor $\alpha$ detectors (bottom). A red line indicates the lower SCA threshold in each spectrum.

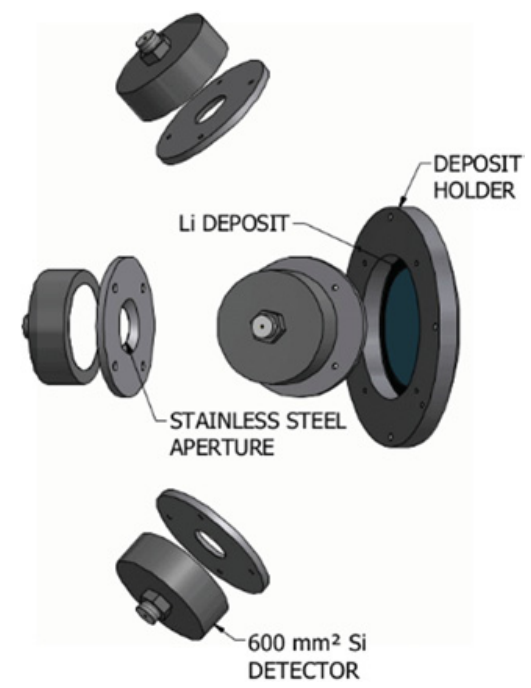

Figure 4. A representation of the layout of the flux monitor used in the BL2 experiment.

the efficiency measurement, namely the solid angle, the deposit areal density, and ${ }^{6} \mathrm{Li}$ cross section, is unnecessary. The structure of the beam is important both in terms of the shape of the beam and its energy. These parameters are inputs to the flux measurement analysis, and the reported efficiency is taken to represent an infinitely narrow beam of thermal neutrons at wavelength $\lambda_{0}$, corresponding to $v_{0}=2200 \mathrm{~m} / \mathrm{s}$. If we represent the wavelength of the monochromatic beam incident on Alpha-Gamma by $\lambda_{A G}$, we can then describe the efficiency of the flux monitor as

$$
\epsilon_{0}=\left(\frac{r_{\gamma, \text { thick }}}{r_{F M}}\right)^{-1}\left(\frac{r_{\alpha, \text { thin }}}{r_{\gamma, \text { thin }}}\right)^{-1}\left(\frac{r_{P u, A G}}{R_{P u, \text { emitted }}}\right)\left(\frac{\lambda_{0}}{\lambda_{A G}}\right) .
$$

The first ratio is determined by the thick run data, the second by the thin runs' data, and the last two by dedicated measurements for the solid angle and the wavelength. Any additional systematic effects provide correction factors which apply multiplicatively to one of these terms; as such, they can be factored out as a multiplicative effect on the total uncertainty. A typical flux monitor calibration campaign requires around ten thick run measurements for a total data collection period of thirty days with a calibrated Alpha-Gamma device. Measurements of the errors from a recent campaign are provided in Table 1. 


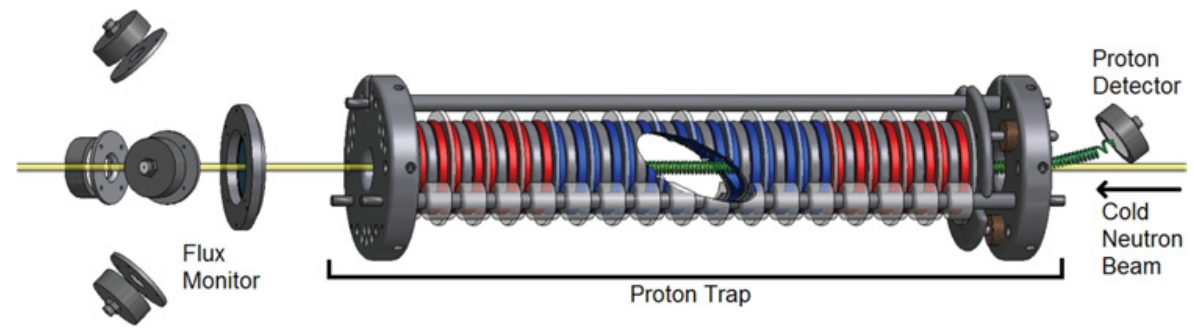

Figure 5. The BL2 proton trap and neutron flux monitor. By modulating the voltages of different numbers of electrodes, the proton trapping volume can be modified.

Table 1. Systematic errors contributing to a recent BL2 flux monitor calibration campaign, from highest to lowest contribution [2].

\begin{tabular}{ll} 
Systematic Effect & $\begin{array}{l}\text { Relative } \\
\text { uncertainty }\end{array}$ \\
\hline $\begin{array}{l}\alpha \text {-source solid angle in Alpha-Gamma } \\
\text { device }\end{array}$ & $2.8 \times 10^{-4}$ \\
$\gamma$ attenuation by thick target & $2.4 \times 10^{-4}$ \\
Neutron beam wavelength & $2.3 \times 10^{-4}$ \\
$\gamma$ attenuation by thin target & $1.2 \times 10^{-4}$ \\
$\lambda / 2$ component in beam & $9.8 \times 10^{-5}$ \\
$\gamma$ signal from absorption in thin target & $9.1 \times 10^{-5}$ \\
substrate & $8.6 \times 10^{-5}$ \\
Dead time (all sources) & $4.0 \times 10^{-5}$ \\
Flux monitor enhancement from neutron & \\
backscatter & $2.8 \times 10^{-5}$ \\
Alpha-Gamma beam spot solid angle to & \\
$\alpha$ detector & $1.8 \times 10^{-5}$ \\
Neutron loss in FM deposit substrate & $1.2 \times 10^{-5}$ \\
Neutron absorption by ${ }^{6} \mathrm{Li}$ & $<1 \times 10^{-5}$ \\
Self-shielding by ${ }^{6} \mathrm{Li}$ deposit & $<1 \times 10^{-5}$ \\
Flux monitor beam spot solid angle & $<1 \times 10^{-5}$ \\
Thick target loss from ${ }^{10} \mathrm{~B}(\mathrm{n}, \gamma)$ reaction & $<1 \times 10^{-5}$ \\
Flux monitor misalignment & $<1 \times 10^{-5}$ \\
\hline Surface scatter from $\mathrm{B}_{4} \mathrm{C}$ & $4.8 \times 10^{-4}$ \\
\hline Total relative systematic uncertainty &
\end{tabular}

\section{Recent and current campaigns}

\subsection{Flux monitor calibration for the BL2 experiment}

The two primary approaches to measuring the neutron lifetime, $\tau_{n}$, one using a cold neutron beam and the other using confined ultracold neutrons, entail completely different sets of systematic effects. This difference makes beam experiments that can achieve a precision comparable to that of the ultracold neutron experiments important in establishing the value of the neutron lifetime. The BL2 beam experiment utilizes a variable length $(L)$ proton trap to trap the protons produced from the neutron beta decay reaction in a beam passing through the experimental volume (see Fig. 5). Trapped protons are detected by grounding the trap electrodes on one end of the trap and utilizing a magnetic field to direct the protons into an offaxis charged particle detector. In such a setup, the number of neutrons in the trap at any given time can be found by integrating the fluence rate $I(v)$ over the velocity, $v$, and the cross sectional area, $A$, of the trapping volume

$$
N_{n}=L \int_{A} d a d v I(v) \frac{1}{v}
$$

The average rate of detected protons is then $\dot{N}_{p}=$ $\tau_{n}^{-1} \epsilon_{p} N_{n}$, where $\epsilon_{p}$ is the proton detector efficiency and $N_{n}$ is given by Eq. (7). The neutron beam flux is measured using the flux monitor described in Sect. 2.2. With the same consideration for the distribution of velocities, the flux monitor detects neutrons at a rate

$$
\dot{N}_{F M}=\epsilon_{0} v_{0} \int_{A} d a d v I(v) \frac{1}{v} .
$$

This assumes that the neutron absorption efficiency in the lithium target varies as $1 / v$, which has been shown to be accurate to better than $0.01 \%$, and that there is no loss of neutrons between the trap and the detector, measured to better than $0.001 \%$ [4]. The above equations can be combined to provide a measurement of the neutron lifetime

$$
\tau_{n}=\frac{L}{\dot{N}_{p}} \frac{\dot{N}_{F M}}{\epsilon_{0}} \frac{\epsilon_{p}}{v_{0}} .
$$

The role of the measurement campaign for the flux monitor on Alpha-Gamma is to determine the efficiency $\epsilon_{0}$ in Eq. (9). The most recent of these campaigns was carried out using a $40 \mu \mathrm{g} / \mathrm{cm}^{2}{ }^{6} \mathrm{LiF}$ target. The efficiency was evaluated as $\left(3.14183 \pm 0.00094_{\text {stat }} \pm 0.00151_{\text {sys }}\right) \times$ $10^{-5}$. This flux monitor will then be installed on the BL2 beamline. The leading systematic uncertainties arise from the statistics in the ${ }^{239} \mathrm{Pu}$ counting and the wavelength measurement; both of these are in the process of being remeasured.

\subsection{U-235 fission product cross section}

By design, the flux monitor calibration does not depend on any externally determined parameters; this makes it an ideal device for measuring neutron cross sections. One of particular interest is the fission cross section of ${ }^{235} \mathrm{U}$, which has been precisely measured to a value of $(582.6 \pm 1.1)$ barns [5]. A $191.7 \mu \mathrm{g} / \mathrm{cm}^{2}$ deposit of ${ }^{235} \mathrm{U}$ has been prepared with $1.6653(17) \%$ doping of ${ }^{234} \mathrm{U}$ on a Si substrate and will be installed into the flux monitor target mount. The ${ }^{234} \mathrm{U}$ alpha rate measured by the flux monitor detectors is used to precisely determine the mass of the ${ }^{234} \mathrm{U}$ in the deposit, which in turn dermines the ${ }^{235} \mathrm{U}$ mass. The cold neutron beam will interact with the deposit and the resulting fission product rate will provide a measure of cross section.

This method of measurement has been calculated to produce a competitive value for the ${ }^{235} \mathrm{U}$ cross section with one week of data. This measurement plan represents a novel, independent method with different systematical uncertainties from other measurements taken by AlphaGamma. As such, agreement of this measurement with 


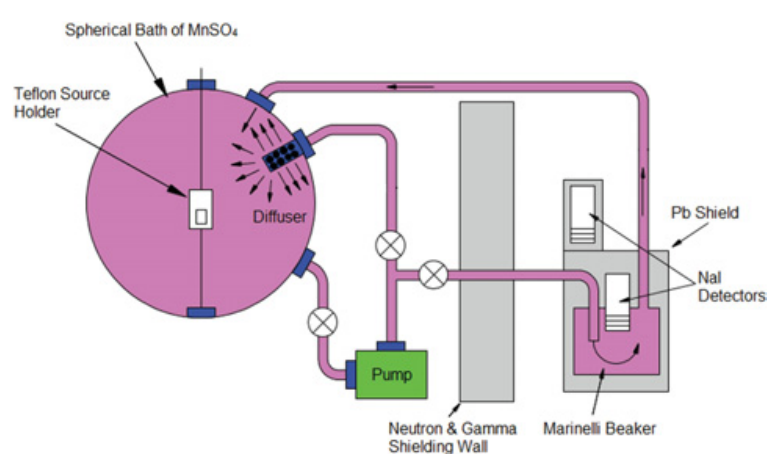

Figure 6. Schematic of the manganese bath including the shielded detector and pumping circuit.

existing values can also be used as a tool in verifying the systematic measurements performed with the AlphaGamma device.

\subsection{NBS-1 recalibration}

The national standard in the United States for determining neutron source emission rates is a $\mathrm{RaBe}$ photoneutron source known as NBS-1. NBS-1 consists of a Pt-Ir capsule containing 1 gram of ${ }^{226} \mathrm{Ra}$ encased in a $4 \mathrm{~cm}$ diameter beryllium sphere [6]. The capsule shields the alpha particles emitted by the radium while permitting passage of the gammas, thus producing a geometrically stable neutron source. The ${ }^{9} \operatorname{Be}(\gamma, n)$ interaction produces neutrons isotropically emitted at a rate of $1.234 \times 10^{6} \pm$ $0.8 \%$ neutrons per second [7]. The calibration of every neutron detector and source in the United States can be traced back to this source and this emission rate measurement. Given the various concerns associated with artifact standards, including the change in emission rate over time and the risk of damage to the source, there is currently an effort underway to remeasure the NBS-1 emission rate using a method with sufficient precision to provide a more robust standard for neutron sources.

A standard method for measuring the strength of isotropic neutron sources is the manganese bath technique [8]. In this method, the neutron source is placed within a cavity inside a sphere filled with an aqueous solution of $\mathrm{MnSO}_{4}$ at $t=0$. The neutrons emitted from the source thermalize in the solution and roughly half of them are captured by natural ${ }^{55} \mathrm{Mn}$ to produce radioactive ${ }^{56} \mathrm{Mn}$, which has a half life of 2.6 hours. This solution is then cycled between the spherical absorption volume and an external, shielded gamma detector, where over time the gamma emission rate asymptotically approaches saturation level, which can be computed from the $i$ th measurement interval as [9]

$$
C_{\infty}^{i}=\frac{C_{i} A(\infty)}{(1 / b) \int_{T_{i}}^{T_{i}+b} A(t-p / 2) e^{-\lambda p / 2} d t},
$$

where $C_{i}$ is the background-subtracted count rate for the $i$ th interval, $b$ is the counting time, $T_{i}$ is the start time of the $i$ th interval, $p$ is the effective pumping time around the detector circuit, and $A(t)$ is the activity in the manganese sulfate bath. $A(\infty)$ represents the asymptotic activity, which is the product of the fraction of source neutrons inducing activity, the source strength at $t=0$, the ratio of the sphere volume to total volume, and the decay constant of the activated manganese $\lambda$. If the source is removed at time $t=T$ when the manganese sulfate solution is at saturation, the decay follows the form

$$
C_{\infty}^{i}=C_{i} \frac{\lambda b A(\infty) e^{\lambda T_{i}}}{A(T)\left[1-e^{-\lambda b}\right]} .
$$

Typically, the measurement of the neutron rate requires knowledge of the efficiency of the detector response relative to the source activity, achievable through coincidence methods and other techniques. At NIST, access to NBS-1 allows the bath to be calibrated to this neutron standard to obtain an absolute measurement. The proposed research utilizes the high precision flux measurement capabilities of Alpha-Gamma to calibrate a manganese bath instead, and then uses that bath to measure the strength of NBS-1. To accomplish this, a smaller version of the standard Mn bath will be installed downstream of Alpha-Gamma. The monochromatic neutron beam will impinge upon the center of the bath via a flight tube inserted in the installation cavity, with total absorption in the manganese sulfate solution. The saturation rate resutling from this will provide a sufficiently precise calibration to increase the NBS-1 neutron rate precision to $0.3 \%$ or better, a factor of three improvement over the current value. A comparison of the emission rate of a transfer source will initially be used to determine the efficacy of this method, and ultimately a recalibration of NBS-1 will be used to establish a new neutron standard. Installation of the small manganese bath on NG-6M is currently underway.

\section{Conclusion}

The Alpha-Gamma device is a powerful tool for precision neutron flux measurement. Its ability to provide sub- $0.1 \%$ flux monitor calibrations is invaluable to the BL2 experiment and is also driving research into other applications. Specifically, preparations for a measurement of the ${ }^{235} \mathrm{U}(\mathrm{n}, \mathrm{f})$ cross section and calibration of a manganese sulfate bath for a new measurement of the NBS-1 neutron source are in progress.

\section{References}

[1] S. Hoogerheide et al., EPJ Web of Conf. 219, 03002 (2019)

[2] A.T. Yue et al., Metrologia 55, 460 (2018)

[3] G.P. Lamaze et al., J. Radioanalytical Nucl. Chem. 123, 551 (1988)

[4] A.T. Yue et al., Phys. Rev. Lett. 111, 222501 (2013)

[5] S.F. Mughabghab, Atlas of Neutron Resonances, http://www.nndc.bnl.gov/atlas/atlasvalues . html (2006)

[6] G. Gamertsfelder, M. Goldhaber, Phys. Rev. 69, 368 (1946)

[7] C.R. Heimbach, J. Testing Evaluation 3 (2002)

[8] J. M. Adams, Nucl. Inst. Meth. in Phys. Res. B 213, 218 (2004)

[9] M. C. Scott, Nucl. Inst. Meth. 82, 237 (1970) 